\title{
196. OBSERVACIONES TAXONÓMICAS ACERCA DE ROSA MOSCHATA HERRM. (ROSACEAE), UNA NOVEDAD PARA LA FLORA ANDALUZA
}

Joel CALVO CASAS

Recibido el 12 de mayo de 2015. aceptado para su publicación el 15 de junio de 2015

Tackling the taxonomy of Rosa moschata Herrm. (Rosaceae), a new species for the Andalusian flora

Palabras clave. Andalucía, Rosaceae, Rosa moschata, Rosa sempervirens, corología.

Keywords. Andalusia, Rosaceae, Rosa moschata, Rosa sempervirens, chorology.

Rosa moschata Herrm. es una especie asilvestrada de origen dudoso (Silvestre \& Montserrat, 1998) que en la Península Ibérica solamente se conocía de algunos puntos del cuadrante noreste (fig. 1). Morfológicamente se parece a $R$. sempervirens L., lo que ha llevado a identificaciones erróneas (Montserrat, 1956; Aymerich 1998, posteriormente corregido por el mismo autor en Aymerich et al. 2008)

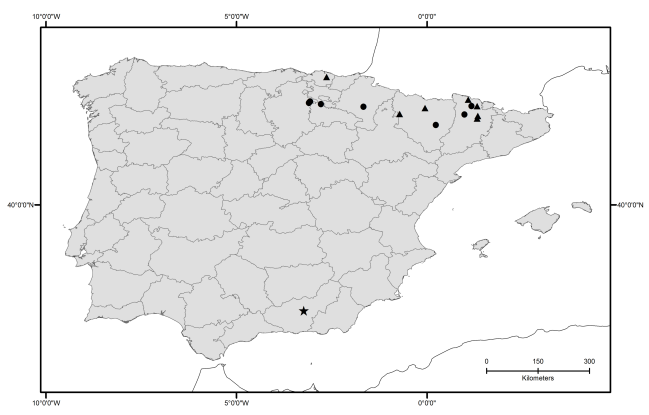

Figura 1. Distribución de Rosa moschata en la Península Ibérica: $(\bullet)$ citas basadas en pliegos de herbario revisados; $(\boldsymbol{\Lambda})$ citas basadas en referencias bibliográficas; $(\star)$ nueva cita para Andalucía. Distribution of Rosa moschata in the Iberian Peninsula: $(\bullet)$ records based on revised herbarium specimens; ( $\mathbf{\Delta}$ ) records based on bibliographic references; ( $\star$ ) new record for Andalusia. $\mathrm{y}$, probablemente, a la infraestimación de las poblaciones de $R$. moschata en nuestro territorio. Estas dos especies se pueden diferenciar por la forma de los sépalos, estructura e indumento de la columna estilar, y por la pilosidad del envés de las hojas jóvenes. Así, Rosa moschata tiene los sépalos anchamente lanceolados, largamente acuminados y al menos los externos con lóbulos marginales (fig. 2-a1), mientras que los de $R$. sempervirens son ovados, agudos o cortamente acuminados, enteros o a veces con pequeños lóbulos marginales (fig. 2-b1). La columna estilar en $R$. sempervirens se caracteriza por estar claramente soldada y tener tricomas abundantes (fig. 2-b2), mientras que en $R$. moschata por lo general los estilos están unidos solo en la base o forman hacecillos, y son casi glabros (con algunos tricomas en la parte inferior) (fig. 2-a2). Otro carácter discriminador de utilidad es la pilosidad del envés de las hojas jóvenes, pues es glabro en $R$. sempervirens y peloso en $R$. moschata, aunque con el tiempo la pilosidad en $R$. moschata desaparece o se mantiene únicamente a lo largo del nervio medio. A menudo se ha utilizado también la consistencia de las hojas para separar las dos especies, describiendo como coriáceas las hojas de $R$. sempervirens. Este carácter

Trabajo financiado por el proyecto Flora iberica (CGL2011-28613-C03-01). 

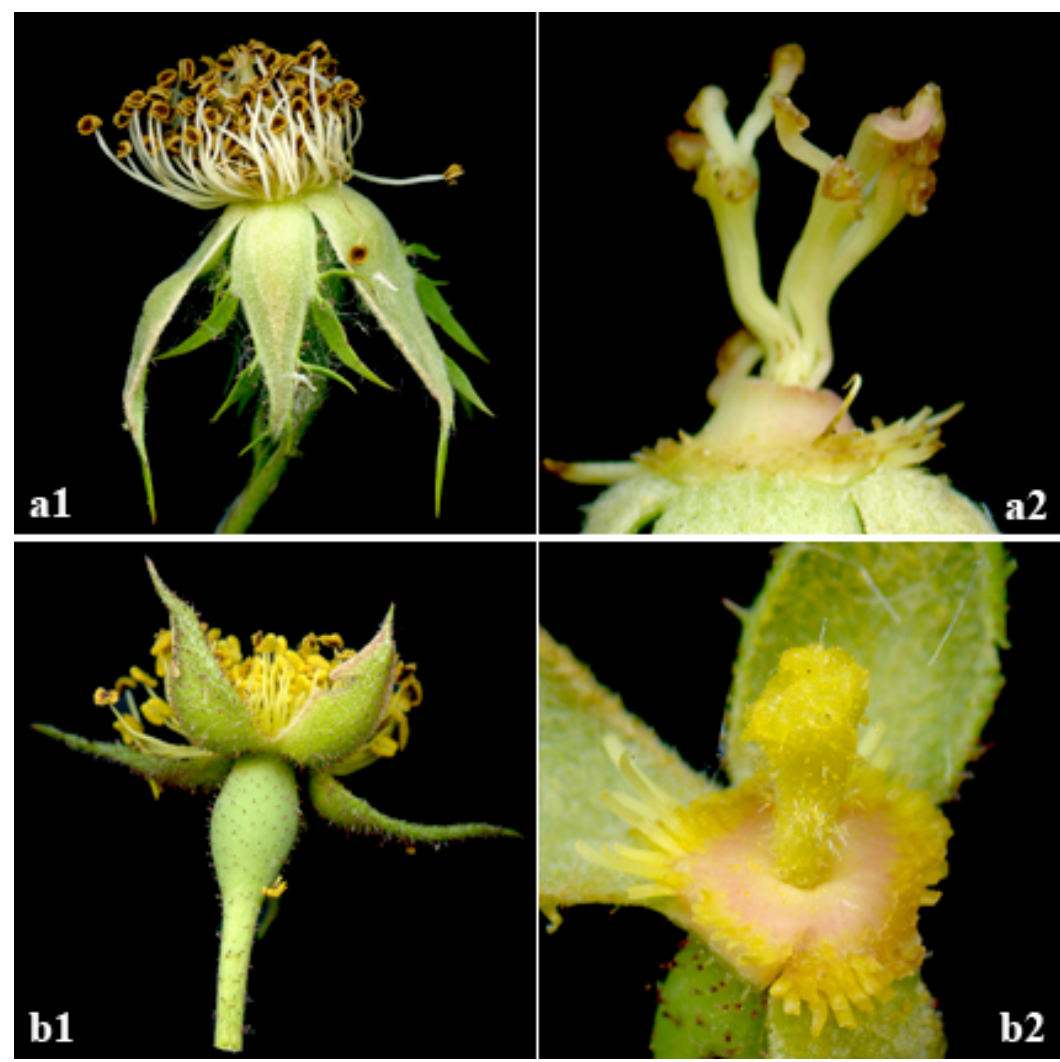

Figura 2. A. Rosa moschata. a1. Detalle de los sépalos; a2. Detalle de la columna estilar. B. Rosa sempervirens. b1. Detalle de los sépalos; b2. Detalle de la columna estilar. Autor: P. Barnola \& J. Vigo / www.floracatalana. net. A. Rosa moschata. a1. Detail of the sepals; a2. Detail of the stylar column. B. Rosa sempervirens. b1. Detail of the sepals; b2. Detail of the stylar column. Author: P. Barnola \& J. Vigo/ www.floracatalana.net.

es muy variable, y aunque efectivamente las formas heliófilas de $R$. sempervirens presentan hojas coriáceas, son frecuentes en esta especie los individuos con hojas algo más endebles (formas esciófilas).

Durante las prospecciones realizadas en Granada durante la primavera de 2014, en el marco del proyecto Flora iberica, encontramos una pequeña población de $R$. moschata en el término municipal de Cortes y Graena (fig. 3). Concretamente se localiza entre Los Baños y La Peza, a $1040 \mathrm{~m}$ de altitud, en un lindero de cultivo al lado de la carretera junto a otras especies de rosa como $R$. micrantha Borrer ex Sm. Con esta especie se elevaría a 13 el número de rosas conocidas hasta el momento en Andalucía según las revisiones de Devesa (1987) y Cueto et al. (2009). Esta cifra no incluye las 6 microespecies del grupo $R$. canina L. reconocidas por los últimos autores.

Material estudiado. ESPAÑA. Álava. La Bastida, [42³5'N 247'W], VII-1928, M. Losa s.n. (MA-56874, sub R. manuelii). Burgos. Ameyugo, río Oroncillo, 42³9'10'N 34'3”'W, 27-VII-2014, J. Calvo \& É. Ross-Nadié JC6699 


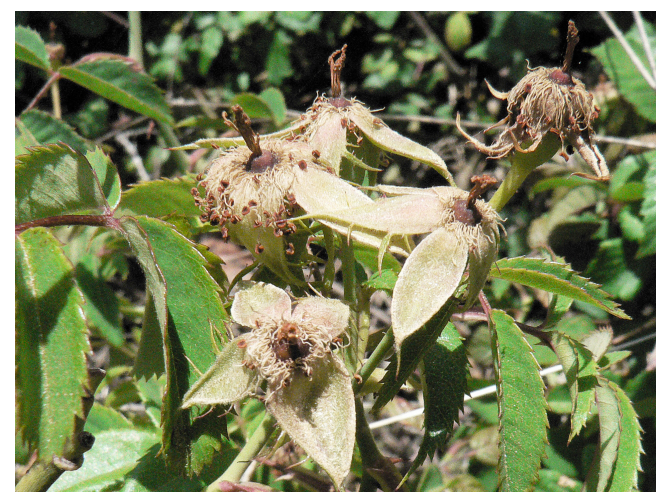

Figura 3. Rosa moschata (pr. Cortes y Graena, Granada). Detalle de los sépalos y columna estilar. Detail of the sepals and stylar column.

(MA-884255). Ameyugo, bois aux bords de l'Oroncillo, [42³9'N 34'W], 15-VII-1916, Hno. Elías 2619 (MA-56871;,MA-155314, P-2721728, P-4155007, sub R. ruscinonensis var. leiostyla). Ameyugo, bords de l'Oroncillo, [4239'N 34'W], 14-VII-1917, Hno. Elías 3256 (MA-56872;,P-4155006, P-2973869, P-4159659, sub R. ruscinonensis var. leiostyla). Pancorbo, [4237'N 3ㅇ'W], 22-VI-1924, Hno. Elías 5164 (MA-56869, MA-56870, sub R. ruscinonensis var. leiostyla). Pancorbo, [4237'N 36'W], 10-VII-1907, Hno. Elias s.n. (MA-57607, sub R. stylosa por C. Pau). Pancorbo, haies de Hontoria, [42 $38^{\circ} \mathrm{N}$ $3^{\circ} 5^{\prime}$ W], VI-1927, M. Losa 6395 (MA-466291, MA-56868, sub R. ruscinonensis). Granada. Cortes y Graena, entre Los Baños y La Peza, pr. Las Viñas, $37^{\circ} 17^{\prime} 35^{\prime \prime} \mathrm{N} 3^{\circ} 14^{\prime} 15^{\prime}$ 'W, 20-VI-2014, J. Calvo \& al. PB1438 (MA-884289, MA-888331). Huesca. Estadilla, alrededores de la casa de Baños, [42 ${ }^{\circ}$ 'N 0¹4'E], 7-VI-1986, G. Montserrat s.n. (MA-535995, sub R. sempervirens por S. Silvestre). Lleida. Escaló, monestir de Sant Pere de Burgal, 42 32'46'N 1'10'01' E, 6-VIII-2014, C. Aedo 22007 (MA-884232). Baix Pallars, a l'estany de Montcortès, [42 ${ }^{\circ} 19^{\prime} \mathrm{N}$ 059'E], 28-VI-2014, P. Barnola \& J. Vigo s.n.
(HGI-22064). Navarra. Tafalla, [42 $31^{\prime} \mathrm{N}$ $\left.1^{\circ} 40^{\prime} \mathrm{W}\right]$, M. Escriche s.n. (MA-56847, sub $R$. sempervirens).

AGRADECIMIENTOS. A Joan Font (HGI) por facilitar material e información de gran utilidad, así como a Josep Vigo y Pere Barnola por prestar amablemente las fotos de detalle de Rosa moschata y $R$. sempervirens.

\section{BIBLIOGRAFÍA}

AYMERICH, P. -1998- Aportació al coneixement florístic del nord de Catalunya. Butl. Inst. Catalana Hist. Nat. 66: 41-57.

AYMERICH, P., I. SORIANO \& J. LLISTOSELLA -2008- Addicions a la flora vascular del Parc Natural del Cadí-Moixeró i de les serres veïnes (Prepirineus Orientals Ibèrics). Acta Bot. Barcinon. 51: 35-47.

CUETO, M., J.L. GONZÁLEZ REBOLLAR \& E. GIMÉNEZ -2009- Rosa L. In: G. Blanca, B. Cabezudo, M. Cueto, C. Fernández López \& C. Morales Torres (eds.), Flora Vascular de Andalucía Oriental 3: 21-26. Consejería de Medio Ambiente, Junta de Andalucía, Sevilla.

DEVESA, J.A. -1987- Rosa L. In: B. Valdés, S. Talavera \& E. Fernández-Galiano (eds.), Flora Vascular de Andalucía Occidental 2: 26-29. Ketres Editora S.A., Barcelona.

MONTSERRAT, P. -1956- Flora de la cordillera litoral catalana (porción comprendida entre los ríos Besòs y Tordera). Collect. Bot. (Barcelona) 5: $1-86$.

SILVESTRE, S. \& P. MONTSERRAT -1998- Rosa L. In: S. Castroviejo (coord. gen.), Flora iberica 6: 143-195. Real Jardín Botánico, CSIC, Madrid.

Dirección del autor. Real Jardín Botánico-CSIC, Plaza Murillo 2, 28014 Madrid, España. calvocasas@ gmail.com 